\title{
A $Q$-MANIFOLD LOCAL-COMPACTIFICATION OF A METRIC COMBINATORIAL $\infty$-MANIFOLD
}

\author{
KATSURO SAKAI
}

\begin{abstract}
Let $K$ be a combinatorial $\infty$-manifold, that is, a countable simplicial complex such that the star of each vertex is combinatorially equivalent to the countable-infinite full simplicial complex. Then the space $|K|_{m}$ with the metric topology is a manifold modeled on the space $\sigma$, where $\sigma$ is the subspace of the Hilbert cube $Q=I^{\omega}$ which consists of all points having at most finitely many nonzero coordinates. In this paper, we give a local-compactification of $|K|_{m}$ which is a $[0,1)$-stable $Q$-manifold containing $|K|_{m}$ as an f.d. cap set.
\end{abstract}

0. Introduction. Let $X$ be a metric space with a metric $d$. A closed subset $A$ of $X$ is called a $Z$-set in $X$ if for each $\varepsilon>0$ and each map $f: I^{n} \rightarrow X, n \in \mathbf{N}$, there is a map $g: I^{n} \rightarrow X \backslash A$ with $d(f, g)<\varepsilon$. An f.d. cap set for $X$ is a subset $M$ of $X$ such that $M=\bigcup_{n=1}^{\infty} M_{n}$ where $M_{1} \subset M_{2} \subset \cdots$ is a tower of finite-dimensional compact $Z$-sets in $X$ and for each $\varepsilon>0$, each $m \in \mathbf{N}$, and each finite-dimensional compact subset $A$ of $X$, there is an $n \in \mathbf{N}$ and an embedding $h: A \rightarrow M_{n}$ such that $h \mid A \cap M_{m}=$ id and $d(h$, id $)<\varepsilon\left[\mathbf{A n}\right.$ and $\left.\mathbf{C h}_{1}\right]$. Let $\sigma$ denote the subspace of the Hilbert cube $Q=I^{\omega}$ which consists of all points having at most finitely many nonzero coordinates. R. D. Anderson [An] showed that $\sigma$ is an f.d. cap set for $Q$ and that for any f.d. cap set $M$ for $Q$ there is a homeomorphism of $Q$ onto itself taking $M$ onto $\sigma$. A Hilbert cube manifold (or $Q$-manifold) is a separable manifold modeled on the Hilbert cube $Q$. A separable manifold modeled on the space $\sigma$ is called a $\sigma$-manifold. T. A. Chapman $\left[\mathbf{C h}_{1}\right]$ proved that any f.d. cap set for a $Q$-manifold is a $\sigma$-manifold and any $\sigma$-manifold can be embedded as an f.d. cap set for a $Q$-manifold and that for any f.d. cap sets $M$ and $N$ for a $Q$-manifold $X$, there is a homeomorphism of $X$ onto itself taking $M$ onto $N$.

Two simplicial complexes are said to be combinatorially equivalent provided they admit simplicially isomorphic subdivisions. A combinatorial $\infty$-manifold is a countable simplicial complex such that the star of each vertex is combinatorially equivalent to the countable-infinite full simplicial complex, namely a $\infty$-simplex $\left[\mathbf{S a}_{1}\right]$. For a simplicial complex $K,|K|$ denotes the underlying set and $|K|_{m}$ denotes the space $|K|$ with the topology induced by the metric

$$
d_{1}(x, y)=\sum_{v \in K^{0}}|x(v)-y(v)|
$$

where $(x(v))_{v \in K^{\circ}}$ and $(y(v))_{v \in K^{\circ}}$ are the barycentric coordinates of $x$ and $y$ respectively. The author $\left[\mathbf{S a}_{2}, \mathbf{S a}_{3}\right]$ showed that any $\sigma$-manifold is homeomorphic to

Received by the editors May 2, 1986.

1980 Mathematics Subject Classification (1985 Revision). Primary 57N20, 57Q15, 54E45, 54F40.

Key words and phrases. Simplicial complex, combinatorial $\infty$-manifold, metric topology, localcompactification, $\sigma$-manifold, $Q$-manifold, $[0,1)$-stable, f.d. cap set, $Z$-set, ANR. 
$|K|_{m}$ for some combinatorial $\infty$-manifold $K$ and that a countable simplicial complex $K$ is a combinatorial $\infty$-manifold if and only if $|K|_{m}$ is a $\sigma$-manifold. For a combinatorial $\infty$-manifold $K$, we give here a local-compactification of $|K|_{m}$ which is a $Q$-manifold containing $|K|_{m}$ as an f.d. cap set.

Through the paper, $K$ always denotes a countable-infinite simplicial complex with vertices $v_{i}, i \in \mathbf{N}$. For each $x \in|K|$, we write $x_{i}=x\left(v_{i}\right), i \in \mathbf{N}$. Identifying $x \in|K|$ with $\left(x_{i}\right)_{i \in \mathbf{N}} \in Q$, we consider $|K| \subset Q$. The topology of $|K|_{m}$ is clearly the subspace topology on $|K|$. Thus $|K|_{m}$ can be considered as a subspace of $Q$. Then $|K|_{m}$ has a local-compactification

$$
\overline{|K|^{Q^{*}}}=\operatorname{cl}_{Q \backslash\{0\}}|K|=\left(\operatorname{cl}_{Q}|K|\right) \backslash\{0\},
$$

where 0 denotes the point $(0,0, \ldots) \in Q$. It is not difficult to see that $\overline{|K|^{Q *}}=$ $|K|$ if and only if $K$ is locally finite. In $\S 1$, we show that $\overline{|K|^{*}}$ an ANR localcompactification of $|K|_{m}$ if $K$ has no principal (maximal) simplex. Our main result is the following which is proved in $\S 2$.

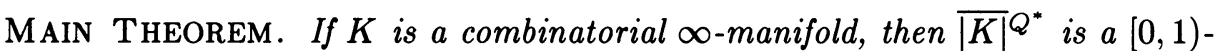
stable $Q$-manifold and $|K|_{m}$ is an f.d. cap set for $\overline{|K|^{Q}}$.

Here we say that a $Q$-manifold $X$ is $[0,1)$-stable if $X \times[0,1)$ is homeomorphic to $X$. T. A. Chapman $\left[\mathbf{C h}_{2}\right]$ showed that $[0,1)$-stable $Q$-manifolds are topologically classified by homotopy type. From this result and the topological uniqueness of f.d. cap sets for a $Q$-manifold, it follows that if two combinatorial $\infty$-manifolds $K$ and

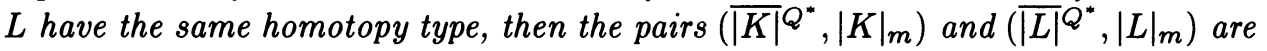
homeomorphic.

The author would like to thank Doug Curtis and V. T. Liem for helpful conversations.

1. The compactification $\operatorname{cl}_{Q}|K|$. We use the metric $d_{Q}$ for $Q$ defined by

$$
d_{Q}(x, y)=\sup _{i \in \mathbf{N}} \min \left\{\left|x_{i}-y_{i}\right|, i^{-1}\right\} .
$$

1.1. LEMMA. If $K$ has no principal simplex, then

$$
\begin{aligned}
\operatorname{cl}_{Q}|K|=\{x \in Q \mid & \text { (1) } \sum_{i=1}^{\infty} x_{i} \leq 1 \text { and } \\
& \text { (2) } \left.v_{i_{1}}, \ldots, v_{i_{n}} \text { span a simplex of } K \text { if } x_{i_{1}}, \ldots, x_{i_{n}} \neq 0\right\} .
\end{aligned}
$$

Proof. It is easy to see that each $x \in \operatorname{cl}_{Q}|K|$ satisfies (1) and (2). (Cf. [Saa Lemma 2.2]. This is valid without hypothesis.) Conversely take $x \in Q$ satisfying (1) and (2). For each $m \in \mathbf{N}$, let

$$
\left\{v_{i_{1}}, \ldots, v_{i_{n}}\right\}=\left\{v_{i} \mid x_{i} \neq 0, i \leq m\right\} .
$$

Then $v_{i_{1}}, \ldots, v_{i_{n}}$ span a simplex of $K$ by (2). Since $K$ has no principal simplex, we have $v_{i_{n+1}} \in K^{0} \backslash\left\{v_{i_{1}}, \ldots, v_{i_{n}}\right\}$ such that $v_{i_{1}}, \ldots, v_{i_{n+1}}$ span a simplex of $K$ and $i_{n+1}>m$. Note $x_{i_{1}}+\cdots+x_{i_{n}} \leq 1$ by (1). We define $y \in|K|$ as follows:

$$
y_{i}= \begin{cases}x_{i_{j}} & \text { if } i=i_{j}, j=1, \ldots, n, \\ 1-\left(x_{i_{1}}+\cdots+x_{i_{n}}\right) & \text { if } i=i_{n+1} \\ 0 & \text { otherwise. }\end{cases}
$$


Since $x_{i}=y_{i}$ for each $i=1, \ldots, m$, we have $d_{Q}(x, y)<m^{-1}$. Therefore $x \in$ $\operatorname{cl}_{Q}|K|$.

1.2. THEOREM. If $K$ has no principal simplex, then $\operatorname{cl}_{Q}|K|$ is an $A R$; hence $\overline{|K|^{Q *}}$ is an $A N R$.

ProOF. Let $\mu: Q \times Q \rightarrow Q$ be a map defined by

$$
\mu(x, y)_{i}=\min \left\{x_{i}, y_{i}\right\}, \quad i \in \mathbf{N} \text {. }
$$

Define a map $\lambda: Q \times Q \times I \rightarrow Q$ as follows:

$$
\lambda(x, y, t)= \begin{cases}(1-2 t) x+2 t \mu(x, y) & \text { if } 0 \leq t \leq \frac{1}{2} \\ (2 t-1) y+(2-2 t) \mu(x, y) & \text { if } \frac{1}{2} \leq t \leq 1\end{cases}
$$

Then $\lambda(x, y, 0)=x, \lambda(x, y, 1)=y$ and $\lambda(x, x, t)=x$ for any $x, y \in Q$ and $t \in I$, that is, $\lambda$ is an equi-connecting map for $Q$. Using Lemma 1.1, it is easily seen that

$$
\lambda\left(\operatorname{cl}_{Q}|K| \times \operatorname{cl}_{Q}|K| \times I\right) \subset \operatorname{cl}_{Q}|K| .
$$

Let $x, y, z \in \operatorname{cl}_{Q}|K|$ such that $d_{Q}(x, z), d_{Q}(y, z)<n^{-1}$, and let $t \in I$. Then for each $i=1, \ldots, n$,

$$
\left|x_{i}-z_{i}\right|,\left|y_{i}-z_{i}\right|<n^{-1} \quad\left(\leq i^{-1}\right)
$$

which implies

$$
\left|\mu(x, y)_{i}-z_{i}\right|=\left|\min \left\{x_{i}, y_{i}\right\}-z_{i}\right|<n^{-1} .
$$

From the definition of $\lambda$, it follows that

$$
\left|\lambda(x, y, t)_{i}-z_{i}\right|<n^{-1} \text { for each } i=1, \ldots, n .
$$

Therefore we have

$$
d_{Q}(\lambda(x, y, t), z)=\sup _{i \in \mathbf{N}} \min \left\{\left|\lambda(x, y, t)_{i}-z_{i}\right|, i^{-1}\right\}<n^{-1} .
$$

This means the $n^{-1}$-neighborhood of $z$ in $\operatorname{cl}_{Q}|K|$ is $\lambda$-convex. By the result of [Du], $\operatorname{cl}_{Q}|K|$ is an $\mathrm{AR}$.

1.3. REMARKS. The hypothesis of Theorem 1.2 is essential. In general $\overline{|K|^{Q *}}$ is not even locally connected. In fact, if $K$ is the cone over the positive integers then $\operatorname{cl}_{Q}|K|$ is homeomorphic to the cone over $\{0\} \cup\{1 / n \mid n \in \mathbf{N}\}$. However, it is not a necessary condition. Let $K$ be the cone over the complex of the half-open interval $[0, \infty)$. Then $\operatorname{cl}_{Q}|K|$ is homeomorphic to a 2 -simplex, hence is an AR.

Under the hypothesis of Theorem 1.2, it can also be shown that the inclusion $|K|_{m} \subset \overline{|K|}^{Q^{*}}$ is a weak homotopy equivalence, therefore a homotopy equivalence (cf. $\left[\mathrm{Sa}_{4}\right.$, Theorem 2.12]).

2. Proof of the Main Theorem and some remarks. First we prove the following

2.1. PROPOSITION. Let $X$ be an ANR local-compactification of a $\sigma$-manifold $M$ and let $d$ be a metric for $X$. If for each $\varepsilon>0$ and each compact subset $A$ of $X$ there is a map $h: A \rightarrow M$ with $d(h$,id $)<\varepsilon$, then $X$ is a $Q$-manifold and $M$ is an f.d. cap set for $X$.

PROOF. H. Toruńczyk [To] characterized $Q$-manifolds as locally compact ANR's $X$ with the disjoint approximation property (i.e., any two maps from $Q$ to $X$ can be 
arbitrarily closely approximated by maps whose images are disjoint). The disjoint approximation property for the $\sigma$-manifold $M$ (cf. [Mo]) and the mapping hypothesis imply that $X$ also has the disjoint approximation property and is therefore a $Q$-manifold.

Next we will show that $M$ is an f.d. cap set for $X$. By [Sa 2 , Lemma 2], $M$ has a strongly universal tower $\left\{M_{i}\right\}_{i \in \mathbf{N}}$ for finite-dimensional compacta such that $M=\bigcup_{i \in \mathbf{N}} M_{i}$ and each $M_{i}$ is a finite-dimensional compact (strong) $Z$-set in $M$ (cf. [CDM]). Then the mapping hypothesis implies that each $M_{i}$ is a $Z$-set in $X$. To verify the absorption property of the tower $\left\{M_{i}\right\}_{i \in \mathbf{N}}$, let $A$ be finite-dimensional compact subset of $X$ and let $m \in \mathbf{N}$ and $\varepsilon>0$. Since $M$ is an ANR, there exists $\delta>0$ such that any map $f: A \cap M_{m} \rightarrow M$ with $d(f$,id $)<\delta$ is $\varepsilon / 3$-homotopic to the inclusion $A \cap M_{m} \subset M$. From the hypothesis, there is a map $h: A \rightarrow M$ with $d\left(h\right.$,id) $<\delta$. Then $h \mid A \cap M_{m}$ is $\varepsilon / 3$-homotopic to the inclusion $A \cap M_{m} \subset M$. By the Homotopy Extension Theorem [Hu, Chapter IV, Theorem 2.2 and its proof], $h$ is $\varepsilon / 3$-homotopic to a map $j: A \rightarrow M$ with $j \mid A \cap M_{m}=$ id. From the strong universality of the tower $\left\{M_{i}\right\}_{i \in \mathbf{N}}$, we have an embedding $g: A \rightarrow M_{n}$ for some $n \geq m$ such that $g\left|A \cap M_{m}=j\right| A \cap M_{m}=$ id and $d(g, j)<\varepsilon / 3$. Then $d(g$, id $) \leq$ $d(g, j)+d(j, h)+d(h$, id $)<\varepsilon$. Hence $M$ is an f.d. cap set for $X$.

PROOF OF THE MAIN THEOREM. To verify the hypothesis of Proposition 2.1 , let $A$ be a compact subset of $\overline{|K|^{Q *}}$ and $\varepsilon>0$. Since $A$ is closed in $Q$ and $0 \notin A$, we have $n \in \mathbf{N}$ and $0<s<1$ such that $n^{-1}<\varepsilon / 3$ and each $x \in A$ has a coordinate $x_{i} \geq s$ for some $i=1, \ldots, n$. Let $L$ be the finite full subcomplex for $K$ with $\left\{v_{1}, \ldots, v_{n}\right\}=L^{0}$, and set

$$
|L|^{*}=\{t x|x \in| L \mid, t \in[s, 1]\} \subset \overline{|K|^{Q^{*}}} .
$$

Define a map $f: A \rightarrow|L|^{*}$ by

$$
f(x)_{i}= \begin{cases}x_{i} & \text { for } i \leq n, \\ 0 & \text { for } i>n .\end{cases}
$$

Then $d_{Q}(f$,id $)<n^{-1}<\varepsilon / 3$. Since $|L|$ is a compact subset of the $\sigma$-manifold $|K|_{m},|L|$ is a $Z$-set in $|K|_{m}$. Hence there is a map $g:|K|_{m} \rightarrow|K|_{m} \backslash|L|$ with $d_{Q}(g$, id $)<\varepsilon / 3$. Since we have a homeomorphism $h:[s, 1] \times|L| \rightarrow|L|^{*}$ defined by $h(t, x)=t x$, we can define a map $g^{*}:|L|^{*} \rightarrow \overline{|K|^{Q^{*}}}$ by $g^{*}(t x)=t g(x)$. Then $d_{Q}\left(g^{*}\right.$, id $) \leq d_{Q}(g$, id $)<\varepsilon / 3$. Observe that

$$
g^{*}\left(|L|^{*}\right) \cap\{t x|x \in| L \mid, t \in[0,1]\}=\varnothing .
$$

This implies that each $x \in g^{*}\left(|L|^{*}\right)$ has a nonzero coordinate $x_{i} \neq 0$ for some $i>n$. From compactness of $g^{*}\left(|L|^{*}\right)$, there exists $m>n$ such that each $x \in g^{*}\left(|L|^{*}\right)$ has a nonzero coordinate $x_{i} \neq 0$ for some $i=n+1, \ldots, m$. Define a map $k: g^{*}\left(|L|^{*}\right) \rightarrow$ $|K|_{m}$ as follows:

$$
k(x)_{i}= \begin{cases}x_{i} & \text { for } i \leq n, \\ \left(1-\sum_{j=1}^{n} x_{j}\right) \cdot x_{i} / \sum_{j=n+1}^{m} x_{j} & \text { for } n<i \leq m, \\ 0 & \text { for } i>m .\end{cases}
$$

Then $d_{Q}(k$, id $)<n^{-1}<\varepsilon / 3$. Thus we have a map $k g^{*} f: A \rightarrow|K|_{m}$. And then

$$
d_{Q}\left(k g^{*} f, \mathrm{id}\right) \leq d_{Q}(k, \mathrm{id})+d_{Q}\left(g^{*}, \mathrm{id}\right)+d_{Q}(f, \mathrm{id})<\varepsilon .
$$


By Proposition 2.1, $\overline{|K|^{Q^{*}}}$ is a $Q$-manifold and $|K|_{m}$ is an f.d. cap set for $\overline{|K|^{Q *}}$.

To verify the $[0,1)$-stability of $\overline{|K|^{Q *}}$, we use Wong's criterion [Wo]. Thus we show that $\overline{|K|^{Q}}{ }^{*}$ is properly contractible to infinity; that is, for each compact subset $A$ of $\overline{|K|^{Q^{*}}}$ there is a proper homotopy $h: \overline{|K|^{Q}} \times I \rightarrow \overline{|K|^{Q}}$ with $h_{0}=$ id and $h_{1}\left(\overline{|K|^{Q}}\right) \subset \overline{|K|^{Q^{*}}} \backslash A$. For any compact subset $A$ of $\overline{|K|^{Q *}}$, we have $n \in \mathbf{N}$ and $0<s<1$ such that

$$
\left\{x \in Q \mid x_{i} \leq s \text { for each } i=1, \ldots, n\right\} \cap A=\varnothing .
$$

Define a homotopy $h: Q \times I \rightarrow Q$ by

$$
h(x, t)=((1-t)+t s) \cdot x .
$$

Then $h_{0}=$ id and $h_{1}(Q) \cap A=\varnothing$. By Lemma $1.1, h\left(\overline{|K|^{Q *}} \times I\right) \subset \overline{|K|^{Q^{*}}}$. Thus we have a proper homotopy $h: \overline{|K|}^{Q^{*}} \times I \rightarrow \mid \overline{\mid K}^{Q^{*}}$ with the desired property. Therefore $\overline{|K|^{Q *}}$ is $[0,1)$-stable.

It is natural to ask the following

2.2. QUESTION. If $\overline{|K|^{Q^{*}}}$ is a $Q$-manifold, then is $K$ a combinatorial $\infty$ manifold?

From the above proof, it follows that $\overline{|K|^{Q^{*}}}$ is $[0,1)$-stable if $\overline{|K|^{Q^{*}}}$ is a $Q$ manifold.

For the countable-infinite full simplicial complex $\Delta^{\infty}, \mathrm{cl}_{Q}\left|\Delta^{\infty}\right|$ is homeomorphic to $Q$ by Keller's Theorem [Ke]. For each $n \in \mathbf{N}$, the identity map of $\operatorname{cl}_{Q}\left|\Delta^{\infty}\right|$ is $n^{-1}$-close to a map $f: \operatorname{cl}_{Q}\left|\Delta^{\infty}\right| \rightarrow\left|\Delta^{\infty}\right|_{m}$ defined by

$$
f(x)_{i}= \begin{cases}x_{i} & \text { for } i \leq n \\ 1-\sum_{i=1}^{n} x_{i} & \text { for } i=n+1 \\ 0 & \text { for } i>n+1\end{cases}
$$

Then as in the proof of Proposition 2.1, $\left|\Delta^{\infty}\right|_{m}$ is an f.d. cap set for $\operatorname{cl}_{Q}\left|\Delta^{\infty}\right|$. Thus the pair $\left(\operatorname{cl}_{Q}\left|\Delta^{\infty}\right|,\left|\Delta^{\infty}\right|_{m}\right)$ is homeomorphic to the pair $(Q, \sigma)$. More generally, the following follows from the Main Theorem.

2.3. COROLlARY. For any contractible combinatorial $\infty$-manifold $K$, the pair $\left(\mathrm{cl}_{Q}|K|,|K|_{m}\right)$ is homeomorphic to the pair $(Q, \sigma)$.

Proof. By the Main Theorem, $\overline{|K|^{Q}}$ is homeomorphic to $Q \times[0,1)$ which is homeomorphic to $Q \backslash\{0\}$. Since $\operatorname{cl}_{Q}|K|$ and $Q$ are the one-point compactifications of $\overline{|K|} Q^{*}$ and $Q \backslash\{0\}$ respectively, they are homeomorphic. Then $\{0\}$ is a $Z$-set in $\operatorname{cl}_{Q}|K|$. Since $|K|_{m}$ is an f.d. cap set for $\overline{|K|^{Q^{*}}}=\left(\operatorname{cl}_{Q}|K|\right) \backslash\{0\}$, it is also an f.d. cap set for $\operatorname{cl}_{Q}|K|$. Thus $\left(\operatorname{cl}_{Q}|K|,|K|_{m}\right)$ is homeomorphic to $(Q, \sigma)$.

However, for a noncontractible combinatorial $\infty$-manifold $K, \operatorname{cl}_{Q}|K|$ is not a $Q$-manifold because $0 \in \operatorname{cl}_{Q}|K|$ and $\{0\}$ is not a $Z$-set in $\operatorname{cl}_{Q}|K|$ (cf. [Saa $]$ ).

\section{REFERENCES}

[An] R. D. Anderson, On sigma-compact subsets of infinite-dimensional spaces, unpublished manuscript.

[Ch $]$ T. A. Chapman, Dense sigma-compact subsets of infinite-dimensional manifolds, Trans. Amer. Math. Soc. 154 (1971), 399-426.

[Ch $\mathbf{C h}_{2} \ldots$, On the structure of Hilbert cube manifolds, Compositio Math. 24 (1972), 329-353. 
[CDM] D. W. Curtis, T. Dobrowolski, and J. Mogilski, Some applications of the topological characterizations of the sigma-compact spaces $l_{f}^{2}$ and $\Sigma$, Trans. Amer. Math. Soc. 284 (1984), 837-846.

[Du] J. Dugundji, Locally equiconnected spaces and absolute neighborhood retracts, Fund. Math. 57 (1965), 187-193.

[Hu] S.-T. Hu, Theory of retracts, Wayne State Univ. Press, Detroit, Mich., 1965.

[Ke] O. H. Keller, Die Homoiomorphie der kompakten konvexen Mengen im Hilbertschen Raum, Math. Ann. 105 (1931), 748-758.

[Mo] J. Mogilski, Characterizing the topology of infinite-dimensional $\sigma$-compact manifolds, Proc. Amer. Math. Soc. 92 (1984), 111-118.

[Sa $\mathbf{S}_{1}$ K. Sakai, Combinatorial infinite-dimensional manifolds and $\mathbf{R}^{\infty}$-manifolds, Topology Appl. (to appear).

[Sa2] _ , On topologies of triangulated infinite-dimensional manifolds, J. Math. Soc. Japan 39 (1987) (in press).

[Sa $\mathbf{S a}_{3}$ _ Simplicial complexes triangulating infinite-dimensional manifolds, preprint.

[Sa4] _ Completions of metric simplicial complexes by using $l_{p}$-norms, preprint.

[To] H. Torunczyk, On CE-images of the Hilbert cube and characterization of Q-manifolds, Fund. Math. 106 (1980), 31-40.

[Wo] R. Y.-T. Wong, Non-compact Hilbert cube manifolds, unpublished manuscript.

Institute of MAThematics, UniVersity of TSUKUBA, SAKURA-MURA, Ibaraki 305, JAPAN (Current address)

Department of Mathematics, Louisiana State University, Baton Rouge, LOUISIANA 70803 